\title{
FLOW OF A RAREFIED GAS WITH THERMAL CREEP IN A CIRCULAR TUBE*
}

\author{
TAtsuo KANKI and SATORU IUCHI \\ Department of Chemical Engineering, Himeji Institute of \\ Technology, Himeji, Japan
}

\begin{abstract}
Rarefied gas channel flow at a uniform temperature has been studied both theoretically and experimentally by many investigators. However, the analytical solution demonstrating explicitly the behavior of the gas flow, especially in the transition flow region where the well-known Knudsen minimum occurs, has not yet been obtained because of the complexity of the momentum transport by molecular collisions. Nor has the gas flow induced by the thermal stress been fully researched.

In this paper, the problem of rarefied gas flow with thermal creep in a circular tube is studied, with special reference to the effect of thermal creep on the gas flow. The solution of the Boltzmann equation with the BGK model is obtained by use of the iteration procedure which uses the slip continuum solution as the first guess.

The volume flow rate of the flow induced by the pressure gradient resulting from the present analysis is in fair agreement with the experimental data in the whole range of the inverse Knudsen number. The solution also gives a fairly good approximation for the thermal creep in consequence of the previous theoretical work.
\end{abstract}

\section{Introduction}

The importance of the study of transport phenomena in a rarefied gas has been growing with the recent development of various operations under vacuum such as drying, evaporating, vaporization coating, distillation and others. In particular, rarefied gas channel flow, which has absorbed much interest of many investigations in this century, surely occurs in various channels by which such equipment is connected with the diffusion pump. The subject also has an immediate connection with gaseous diffusion through porous media.

The rarefied gas flow problem was studied first by Knudsen ${ }^{11)}$, and later, on the basis of the uniform gas kinetic theory, by Adzumi ${ }^{1)}$, Scott and Dullien ${ }^{15)}$, Dong $^{4)}$, and many others. Recently, theoretical work based on the nonuniform gas kinetic theory was carried out by Takao $^{16)}$, Cercignani and Sernagiotto ${ }^{3)}$, $\mathrm{Liu}^{12)}$, Sone and Yamamoto ${ }^{14)}$, Namba, Shinagawa, and Kawamura ${ }^{13)}$, Kanki and $\mathrm{Iuchi}^{8)}$, and others. However, no solution which gives results in good agreement with the experimental data in the whole flow region, especially in the transition flow region, has yet been obtained.

In practice, vacuum equipment is mostly operated

\footnotetext{
* Received on May 7, 1973

Presented at the 37 th Annual Meeting (at Tokyo, April 4, 1972) and at the Autumn Meeting (at Osaka, Oct. 6, 1971) of The Soc. of Chem. Engrs., Japan

于671-22 姫路市書写2167

姫路工業大学産業機械工学科 神吉達夫
}

under conditions of nonuniform temperature. Therefore, the gas is considered to be in the state of nonuniform temperature, and gas flow induced by thermal stress surely occurs. However, thermal creep flow, which is not considered in the framework of classical hydrodynamics, has not been fully researched.

In this paper, rarefied gas flow resulting from both a pressure and temperature gradient in a circular tube is studied. The chief purposes of this paper are threefold: first, to obtain an analytical solution of the BoltzmannKrook equation to the problem; second, to have the gas flow rates of both the Poiseuille flow and thermal creep as an explicit function of the inverse Knudsen number; and third, to examine the effects of thermal creep on rarefied gas flow.

Sone and Yamamoto ${ }^{14)}$ formulated the same problem and obtained the solution of the Boltzmann-Krook equation. The solution, however, does not give appropriate results in the transition flow region, because of the slow convergence in that region. So we tried to solve the problem by use of another method, based on the Willis iteration procedure ${ }^{17}$ ), in which the continuum hydrodynamical solution including slip flow, in place of the free molecular approach (Willis used the free molecular solution), is used as the first guess.

The present solution of the gas flow induced by the pressure gradient (Poiseuille flow) is compared with the experimental data, Knudsen's semi-empirical formula and the numerical solution of Cercignani and Cernagiotto ${ }^{3)}$. The solution is in fair agreement with those results in the whole range of the inverse Knudsen number $\delta$. As to the thermal creep, the comparison between the present solution and the previous numerical 


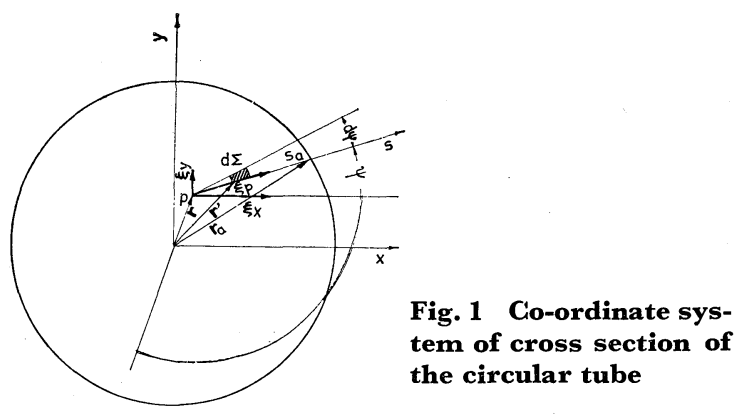

solution $^{8)}$ is also given. The solution gives fairly good results in the whole range of $\delta$.

Finally, the problem of the thermal transpiration; the static state of no gross flow in which dynamical equilibrium is established and a pressure gradient is supported by an applied temperature gradient along the channel is discussed.

\section{Analysis}

\section{1 Formulation of the basic equation}

Let us consider the Cartesian co-ordinate system $\boldsymbol{x}=(x, y, z)$, with $z$ as the axis of symmetry of the circular tube whose wall is maintained by the nonuniform temperature dependent on $z$ only; $T=T_{o}$ $\left[1+\kappa_{T}\left(z / r_{o}\right)\right]$, where $\kappa_{T}$ is a dimensionless temperature gradient (defined by $\kappa_{T}=r_{o} T_{o}^{-1} d T / d z$ ), which is assumed small relative to unity. The pressure along the channel is $p=p_{o}\left[1+\kappa_{P}\left(z / r_{o}\right)\right]$, where $\kappa_{P}$ is a dimensionless pressure gradient written as $\kappa_{P}=r_{o}$ $\times p_{o}^{-1} d p / d z$ and small relative to unity. We study the steady flow of gas resulting from both the pressure and temperature gradient.

In the analysis, we use the Boltzmann equation with the Bhatnagar-Gross-Krook model. Following the obvious linearized approach due to Gross et al. ${ }^{6}$, we have the linearized Boltzmann equation as follows:

$$
\begin{gathered}
\xi_{x} \frac{\partial \phi}{\partial x}+\xi_{y} \frac{\partial \phi}{\partial y}+\xi_{z} \frac{\partial \phi}{\partial z}=\sigma[-\phi+\nu+2 h \xi \hat{q} \\
f=f_{o}(1+\phi) \\
f_{o}=\left(\frac{h}{\pi}\right)^{3 / 2} \exp \left[-h\left(\xi^{2}-3 / 2\right)\right] \\
\nu=\int \phi f_{o}^{2} d \xi \\
\left.\left.\boldsymbol{q}=\int \boldsymbol{\xi} \phi f_{o}^{2}+\xi_{z}^{2}\right)\right] \\
(\nu+\tau)=\frac{2 h}{3} \int \xi^{2} \phi f_{o} d \xi \\
h=m / 2 k T
\end{gathered}
$$

where $f$ is the velocity distribution function of the gas molecules, $\phi$ the relative change of $f, \xi$ the molecular velocity vector, $\sigma$ the collision frequency related to the mean free path $\lambda$ as $\sigma=2 / \lambda(\pi h)^{1 / 2}, \nu$ the relative change of the molecular number density, $\tau$ the relative change of the absolute temperature $T$, and $\boldsymbol{q}\left(q_{x}, q_{y}, q_{z}\right)$ is the gas velocity.

We here assume that the gas molecules impinging on the channel wall reflect diffusely; the reflected molecules have a Maxwellian distribution characterized by the wall temperature. Then the boundary condition for the problem can be written as

$$
\phi=\frac{\kappa_{P}-\kappa_{T}}{r_{o}} z+\kappa_{T} \frac{z}{r_{o}}\left(h \xi^{2}-3 / 2\right) \text { at }\left(x^{2}+y^{2}\right)^{1 / 2}=r_{o}
$$

where we used the relations

$$
\nu=\left(\kappa_{P}-\kappa_{T}\right) z / r_{o} \quad \tau=\kappa_{T} z / r_{o}
$$

We try to find the solution in the following form:

where

$$
\phi=\phi_{o}(\xi) z / r_{o}+\phi_{1}(x, y, \xi)
$$

$$
\phi_{o}=\kappa_{P}+\kappa_{T}\left(h \xi^{2}-5 / 2\right)
$$

Equation(3) is compatible with the governing equation and the boundary condition ${ }^{14)}$.

We further assume very slow flow; the gas velocity is independent of $z$-direction and is a function of $x$ and $y$ only.

Introducing Eq.(3) into Eq.(1), we have

$$
\xi_{P} \frac{\partial \phi_{1}}{\partial s}+\sigma \phi_{1}=\xi_{z}\left[2 \sigma h q_{z}-\frac{\kappa_{P}}{r_{o}}-\frac{\kappa_{T}}{r_{o}}\left(h \xi^{2}-5 / 2\right)\right]
$$

with the boundary condition

$$
\phi_{1}=0 \quad \text { at }\left(x^{2}+y^{2}\right)^{1 / 2}=r_{o}
$$

where $\xi_{p}^{2}=\xi_{x}^{2}+\xi_{y}^{2}$ and $s$ is a co-ordinate along the $\xi_{p}$ direction as shown in Fig. 1.

Since the dimensionless pressure and temperature gradient $\kappa_{P}$ and $\kappa_{T}$ are independent of each other, the first perturbation term $\phi_{1}$ can be expressed by the linear combination of $\kappa_{P}$ and $\kappa_{T}$ as

$$
\phi_{1}=\phi_{1 P} \kappa_{P}+\phi_{1 T} \kappa_{T}
$$

where $\phi_{1 P}$ and $\phi_{1 T}$ are respectively the relative change of the velocity distribution function directly related to the Poiseuille flow and that to the thermal creep.

Using the definition of the gas velocity in the $z$ direction given by

$$
q_{z}(r)=\frac{h^{3 / 2}}{\pi^{3 / 2}} \iiint \xi_{z} \phi_{1}(x, y, \xi) \exp \left(-h \xi^{2}\right) d \xi_{x} d \xi_{y} d \xi_{z}
$$

Equation (4) further becomes

$$
\begin{aligned}
& \xi_{p} \frac{\partial \phi_{1 P}}{\partial s} \kappa_{P}+\xi_{p} \frac{\partial \phi_{1 T}}{\partial s} \kappa_{T}+\sigma\left(\phi_{1 P} \kappa_{P}+\phi_{1 T} \kappa_{T}\right) \\
&= \xi_{z}\left[2 \sigma \frac{h^{5 / 2}}{\pi^{3 / 2}} \int \xi_{z} \phi_{1 P} \exp \left(-h \xi^{2}\right) d \xi \cdot \kappa_{P}+2 \sigma \frac{h^{5 / 2}}{\pi^{3 / 2}}\right. \\
&\left.\cdot \int \xi_{z} \phi_{1 T} \exp \left(-h \xi^{2}\right) d \xi \cdot \kappa_{T}-\frac{\kappa_{P}}{r_{o}}-\frac{\kappa_{T}}{r_{o}}\left(h \xi^{2}-5 / 2\right)\right]
\end{aligned}
$$

with the boundary conditions

$$
\begin{array}{ll}
\phi_{1 P}=0 & \text { at } s=s_{a} \\
\phi_{1 T}=0 & \text { at } s=s_{a}
\end{array}
$$


Equation (8) can be divided into the following two forms:

$$
\begin{gathered}
\frac{\partial}{\partial \bar{s}} \phi_{1 P}+\frac{1}{\bar{\xi}_{p}} \delta \phi_{1 P}=\frac{\bar{\xi}_{z}}{\bar{\xi}_{p}} \frac{2 \delta}{\pi^{3 / 2}} \int \bar{\xi}_{z} \phi_{1 P} \exp \left(-\bar{\xi}^{2}\right) d \bar{\xi}-\frac{\bar{\xi}_{z}}{\bar{\xi}_{p}} \\
\frac{\partial}{\partial s} \phi_{1 T}+\frac{1}{\bar{\xi}_{p}} \delta \phi_{1 T}= \\
=\frac{\bar{\xi}_{z}}{\bar{\xi}_{p}} \frac{2 \delta}{\pi^{3 / 2}} \int \bar{\xi}_{z} \phi_{1 T} \exp \left(-\bar{\xi}^{2}\right) d \bar{\xi} \\
-\frac{\bar{\xi}_{z}}{\bar{\xi}_{p}}\left(\bar{\xi}^{2}-5 / 2\right)
\end{gathered}
$$

where $\bar{s}=s / r_{o}, \delta=h^{1 / 2} \sigma r_{o} \quad\left(=2 r_{o} / \pi^{1 / 2} \lambda\right)$, and $\bar{\xi}=h^{1 / 2} \xi$.

Integrating Eqs. (10-1) and (10-2) with respect to $\bar{s}$ under the boundary conditions in Eqs. (9-1) and (9-2), we have

$$
\begin{aligned}
\phi_{1 P}(\overline{\boldsymbol{r}}, \overline{\boldsymbol{\xi}})= & \int_{\bar{s}_{a}}^{\bar{s}}\left[2 \delta \frac{\bar{\xi}_{z}}{\bar{\xi}_{p}} \frac{1}{\pi^{3 / 2}} \int \bar{\xi}_{z} \phi_{1 P}\left(\overline{\boldsymbol{r}}^{\prime}, \overline{\boldsymbol{\xi}}\right) \exp \left(-\bar{\xi}^{2}\right) d \overline{\boldsymbol{\xi}}\right. \\
& \left.-\frac{\bar{\xi}_{z}}{\bar{\xi}_{p}}\right] \exp \left[-\frac{\delta}{\bar{\xi}_{p}}\left|\overline{\boldsymbol{r}}-\overline{\boldsymbol{r}}^{\prime}\right|\right] d \bar{s}^{\prime} \\
\phi_{1 T}(\overline{\boldsymbol{r}}, \overline{\boldsymbol{\xi}})= & \int_{\bar{s}_{a} L}^{\bar{s}}\left[2 \delta \frac{\bar{\xi}_{z}}{\bar{\xi}_{p}} \frac{1}{\pi^{3 / 2}} \int \bar{\xi}_{z} \phi_{1 T}(\overline{\boldsymbol{r}}, \overline{\boldsymbol{\xi}}) \exp \left(-\overline{\boldsymbol{\xi}}^{2}\right) d \overline{\boldsymbol{\xi}}\right. \\
& \left.-\frac{\bar{\xi}_{z}}{\bar{\xi}_{p}}\left(\bar{\xi}_{p}^{2}+\bar{\xi}_{z}^{2}-5 / 2\right)\right] \cdot \exp \left[-\frac{\delta}{\bar{\xi}_{p}}\left|\overline{\boldsymbol{r}}-\overline{\boldsymbol{r}}^{\prime}\right|\right] d \bar{s}^{\prime}
\end{aligned}
$$

where $\overline{\boldsymbol{r}}$ is the radius vector given by $\overline{\boldsymbol{r}}=\left(x / r_{o}, y / r_{o}\right)$ and $\bar{s}$ corresponds to $\overline{\boldsymbol{r}}$.

\section{2 Solution}

We try to find the solutions of Eqs. (11) and (12) by approaching from the continuum side on the basis of the Willis iteration procedure. We use the hydrodynamical solution as the first guess.

The gas velocity with slip induced by the pressure gradient is given by ${ }^{10)}$

$$
\bar{q}_{z P}(\bar{r})=\frac{\pi}{16} \delta\left(1-\bar{r}^{2}+4 / \pi^{1 / 2} \delta\right)
$$

which holds at $\delta \gg 1$.

As to the thermal creep, the gas velocity is certainly considered to be zero in the continuum flow region, because flow by thermal stress is not a continuum but a thermal diffusive flow process. Thus, the asymptotic solution for the region of $\delta \gg 1$ can be written as

$$
\bar{q}_{z T}=0
$$

Substituting Eq.(13) into the integral term $\int \bar{\xi}_{z} \phi_{1 P}$ $\exp \left(-\bar{\xi}^{2}\right) \cdot d \overline{\boldsymbol{\xi}} / \pi^{3 / 2}$ in Eq.(11), Eq.(14) into the term $\int \bar{\xi}_{z} \phi_{1 T} \exp \left(-\bar{\xi}^{2}\right) d \xi / \pi^{3 / 2}$ in Eq.(12), multiplying the resulting equations by $\bar{\xi}_{z}$, and integrating over the appropriate velocity range, we have the gas velocity of the Poiseuille flow and that of the thermal creep as follows;

Poiseuille velocity:

$$
\bar{q}_{z P}(\bar{r})=\frac{1}{\pi^{3 / 2}} \int_{\Sigma} \int_{-\infty}^{\infty} \int_{0}^{\infty}|J| \bar{\xi}_{z}\left[2 \delta \frac{\bar{\xi}_{z}}{\bar{\xi}_{p}} \bar{q}\left(\bar{r}^{\prime}\right)-\frac{\bar{\xi}_{z}}{\bar{\xi}_{p}}\right]
$$

$$
\begin{aligned}
& \cdot \exp \left(-\bar{\xi}_{z}^{2}\right) \cdot \frac{\exp \left\{-\left[\frac{\delta}{\bar{\xi}_{p}}\left(\left|\overline{\boldsymbol{r}}-\overline{\boldsymbol{r}}^{\prime}\right|\right)+\bar{\xi}_{p}^{2}\right]\right\}}{\left|\overline{\boldsymbol{r}}-\overline{\boldsymbol{r}}^{\prime}\right|} \\
& \cdot d \bar{\xi}_{p} d \bar{\xi}_{z} d \Sigma
\end{aligned}
$$

thermal creep velocity:

$$
\begin{aligned}
\bar{q}_{z T}(\bar{r})= & \frac{1}{\pi^{3 / 2}} \int_{\Sigma} \int_{-\infty}^{\infty} \int_{0}^{\infty}|J| \bar{\xi}_{z}\left[\frac{\bar{\xi}_{z}}{\bar{\xi}_{p}}\left(\bar{\xi}_{z}^{2}+\bar{\xi}_{p}^{2}-5 / 2\right)\right] \\
& \cdot \exp \left(-\bar{\xi}_{z}^{2}\right) \cdot \frac{\exp \left\{-\left[\frac{\delta}{\bar{\xi}_{p}}\left(\left|\overline{\boldsymbol{r}}-\overline{\boldsymbol{r}}^{\prime}\right|\right)+\bar{\xi}_{p}^{2}\right]\right\}}{\left|\overline{\boldsymbol{r}}-\overline{\boldsymbol{r}}^{\prime}\right|} \\
& \cdot \overline{d \xi_{p}} d \bar{\xi}_{z} d \Sigma
\end{aligned}
$$

where $|J|$ is the Jacobian given by $|J|=\xi_{p}$ and $\sum$ is the cross-section area of the circular tube.

After integration of Eqs.(15) and (16) with respect to $\xi_{p}, \xi_{z}$, and $\sum$, the gas velocity of the Poiseuille flow and that of the thermal creep reduce to

$$
\begin{aligned}
\bar{q}_{z P}(\vec{r})= & \frac{1}{\pi} \int_{0}^{2 \pi}\left\{-\left[\frac{\pi}{16} \delta\left(1-\bar{r}^{2}\right)+\frac{\pi^{1 / 2}}{4}\right]\right\} \cdot\left[\frac{1}{2}\right. \\
& \left.-T_{1}(\chi)\right] d \psi+\frac{1}{\pi} \int_{0}^{2 \pi}\left\{\frac { \pi } { 1 6 \delta } \left[\chi^{2} T_{1}(\chi)+2 \chi T_{2}(\chi)\right.\right. \\
& \left.+2 T_{3}(\chi)-1\right]+\frac{\pi}{8} r\left[-\chi T_{1}(\chi)-T_{2}(\chi)\right. \\
& \left.\left.+\frac{\pi^{1 / 2}}{4}\right] \cos \psi\right\} d \psi \\
\bar{q}_{z T}(\tilde{r})= & \frac{1}{2 \delta \pi} \int_{0}^{2 \pi}\left[T_{1}(\chi)-T_{3}(\chi)\right] d \psi
\end{aligned}
$$

where $T_{n}(\chi)=\int_{0}^{\infty} \zeta^{n} \exp \left(-\zeta^{2}+\frac{\chi}{\zeta}\right) d \zeta$

with $\chi=\delta\left(\bar{r} \cos \psi+\sqrt{\left.1-\bar{r}^{2} \sin ^{2} \psi\right)}\right.$

The volume flow rate of the Poiseuille flow and that of the thermal creep are respectively

$$
\begin{aligned}
& \bar{Q}_{P}=\int_{0}^{1} 2 \pi \bar{r} \bar{q}_{z P}(\bar{r}) d \bar{r} \\
& \bar{Q}_{T}=\int_{0}^{1} 2 \pi \bar{r} \bar{q}_{z T}(\bar{r}) d \bar{r}
\end{aligned}
$$

\section{Discussion}

The gas flow rate of the Poiseuille flow calculated from the present solution is compared with Knudsen's semi-empirical formula, the numerical solution of the BGK equation ${ }^{3)}$, and the experimental data $^{7)}$ in Fig. 2. The solution gives results which are in fair agreement with the data in the whole range of the inverse Knudsen number, and is closely related to both Knudsen's formula and Cercignani's solution. The asymptotic solution of the free molecular flow by Willis iteration procedure expressed as

$$
\bar{Q}_{P}=4 \pi^{1 / 2} / 3+\pi \delta \ln \delta / 2
$$

(see Appendix 1) is also shown in Fig. 2. This perfectly agrees with Smoluchowski's free molecular solution, $\bar{Q}_{P}=4 \pi^{1 / 2} / 3$, when $\delta \rightarrow 0$. The broken line with three 


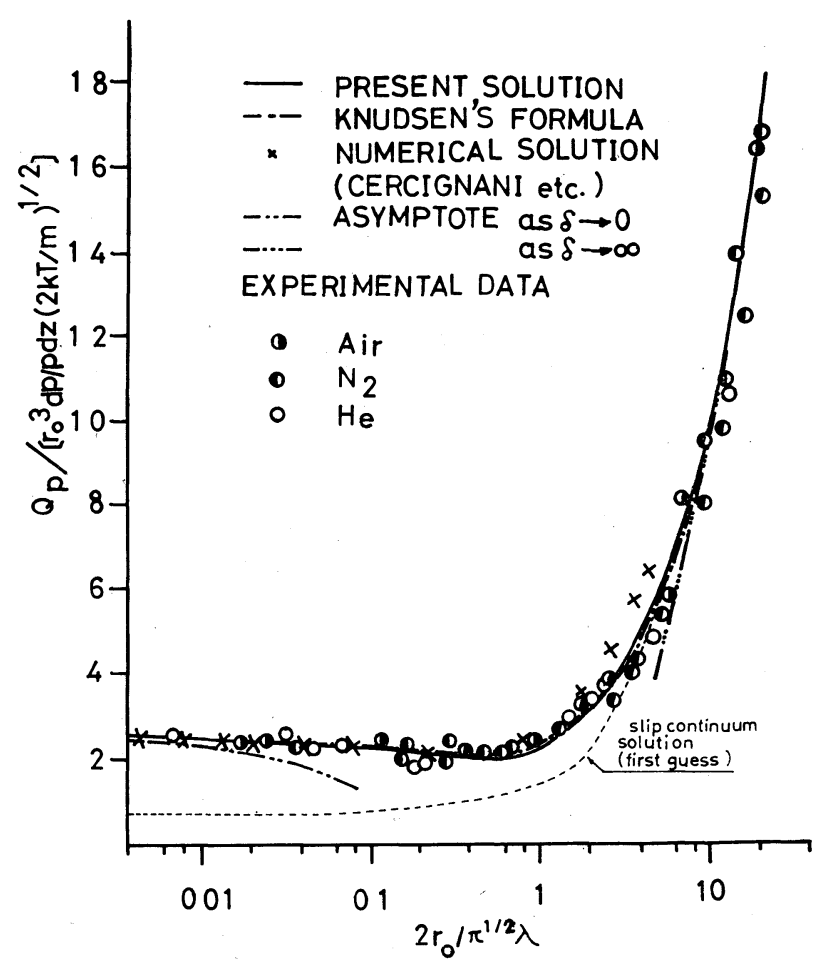

Fig. 2 Volume flow rate of the Poiseuille flow

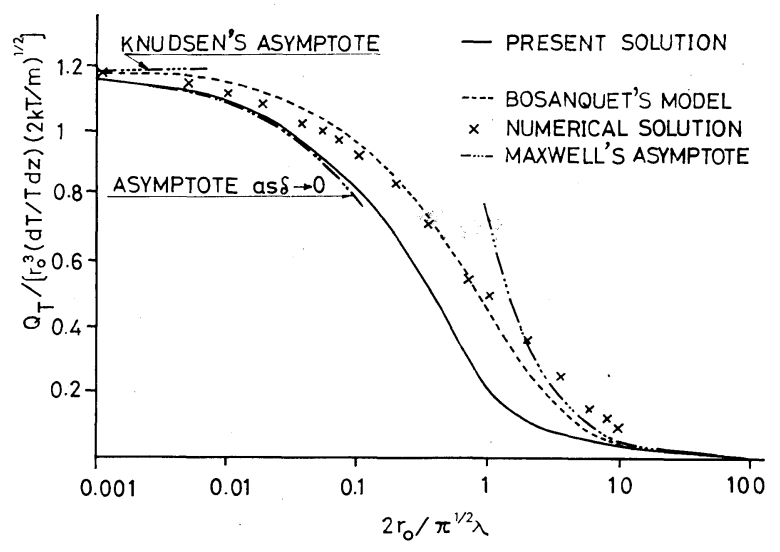

Fig. 3 Volume flow rate of the thermal creep

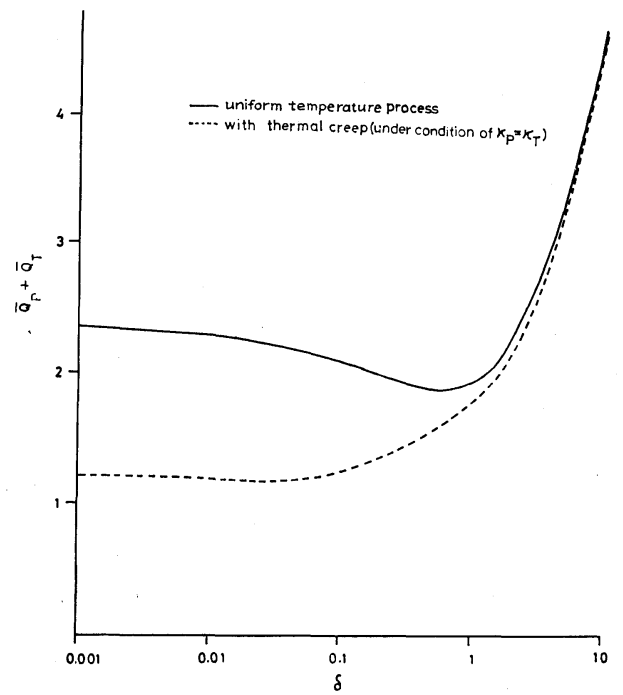

Fig. 4 Effect of the thermal creep on the Poiseuille flow rate under the condition of $\boldsymbol{\kappa}_{P}=\boldsymbol{\kappa}_{T}$

\begin{tabular}{llllll}
\hline \multicolumn{1}{c}{ Table 1 } & \multicolumn{2}{c}{ Value of $\boldsymbol{\delta}$ where the minimum occurs } \\
\multicolumn{1}{c}{ Authors } & Takao $\begin{array}{c}\text { Cercignani } \\
\text { Sernagiotto }\end{array}$ & Knudsen & $\begin{array}{c}\text { Experi- } \\
\text { ment }\end{array}$ & $\begin{array}{c}\text { Present } \\
\text { work }\end{array}$ \\
\hline $\begin{array}{l}\text { Collision } \\
\text { model; }\end{array}$ & BGK & BGK & - & - & BGK \\
$\delta$ for $Q_{P \text { min }} ;$ & 0.21 & 0.3 & 0.79 & $0.2-2$ & 0.6 \\
$Q_{P \min } ;$ & 1.23 & 1.37 & 2.04 & $1.8-2.1$ & 1.89 \\
\hline
\end{tabular}

points in the figure shows the continuum-slip solution. The present solution closely approaches the free molecular solution as $\delta \rightarrow 0$, and the laminar continuum solution as $\delta \rightarrow \infty$. As seen in the figure, the solution predicts a minimum at $\delta \simeq 0.6$. The minimum was found first by Knudsen, and has been widely discussed by many authors. For comparison, results on the minimum flow rate from recent investigations are illustrated in Table 1.

The flow rate of the thermal creep resulting from the present analysis, the previous numerical solution of the BGK equation, ${ }^{8)}$ Bosanquet's model, ${ }^{2)}$ Maxwell's asymptotic solution ${ }^{10)}$, and Knudsen's free molecular solution ${ }^{11)}$ are shown in Fig. 3. The broken line with two points shows the solution by Willis iteration procedure, which is written as

$$
\bar{Q}_{T}=2 \pi^{1 / 2} / 3+\pi \delta \ln \delta / 2
$$

(see Appendix 2). This equation represents the behavior of the thermal creep in the free molecular flow region. As seen in the figure, the present solution is in fair agreement with Eq.(22) at $\delta<0.1$. It converges on Knudsen's asymptote as $\delta \rightarrow 0$, and approaches Maxwell's asymptotic solution as $\delta \rightarrow \infty$.

Supposing that the channel wall were maintained by a given temperature gradient and that the gas flow were performed in a nonuniform temperature, it would be easily inferred that the thermal creep has a profound influence upon the ordinary gas flow. The volume flow rate calculated by taking the effect of the thermal creep into account (for the case of $\kappa_{P}=\kappa_{T}$ ) is shown in Fig. 4. The figure shows that the effect appears slight in the transition region, becomes larger as the inverse Knudsen number decreases, and reduces the flow rate by half in the free molecular flow region. The effect naturally becomes less as $\delta$ becomes larger, and vanishes when $\delta \rightarrow \infty$. Interestingly, $\delta$ for the Knudsen minimum flow rate $Q_{\min }$ is moved from 0.6 to 0.02 .

As a numerical example, for air at $T=186^{\circ} \mathrm{K}$, $p=0.01 \mathrm{~mm} \mathrm{Hg}$; hence if $r_{o}=0.5 \mathrm{~cm}$ and the length of the tube is $10 \mathrm{~cm}$, we have $\delta=1.16$, where we used the relation: $\lambda=4.864 \times 10^{-3} / p$. If, in particular, we make the temperature difference $\Delta T=214^{\circ} \mathrm{K}$ (for instance $T_{1}=293^{\circ} \mathrm{K}, T_{2}=79^{\circ} \mathrm{K}$ ) and the pressure difference $\Delta p=0.002 \mathrm{mmHg}$ (for instance $p_{1}=0.011 \mathrm{mmHg}$, $p_{2}=0.009 \mathrm{mmHg}$ ), we have $\kappa_{P}=0.02, \kappa_{T}=0.115$, and accordingly $\kappa_{P} Q_{P}=0.04$ from Fig. $2, \kappa_{T} Q_{T}=0.023$ from Fig. 3. If then we use the value for the sonic velocity $(2 \mathrm{kT} / \mathrm{m})^{1 / 2}=3.25 \times 10^{4} \mathrm{~cm} / \mathrm{sec}$, we have $Q_{P}=$ $3.25 \times 10^{2} \mathrm{~cm}^{3} / \mathrm{sec}$ and $Q_{T}=1.87 \times 10^{2} \mathrm{~cm}^{3} / \mathrm{sec}$, and for the gross flow rate $Q=1.38 \times 10^{2} \mathrm{~cm}^{3} / \mathrm{sec}$. In this 


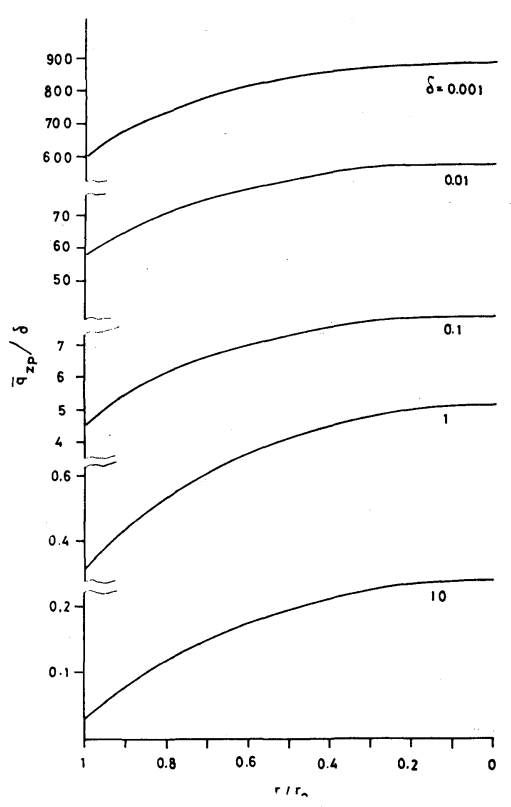

Fig. 5 Gas velocity profile of the Poiseuille flow

case, the volume flow rate due to thermal creep is 57.5 percent of that of the Poiseuille flow.

The relationship of the gas velocity profiles of the flow induced by the pressure gradient and $r / r_{o}$ with some typical inverse Knudsen number is shown in Fig. 5. The gas velocity has a parabolic profile and has a finite value called the slip velocity at the channel wall. The parabolic velocity profile becomes more gentle as the inverse Knudsen number decreases. As $\delta$ increases, on the contrary, the velocity converges on the well-known Hagen Poiseuille parabolic velocity profile.

The thermal creep velocity also has a gentle parabolic profile slightly similar to that of the Poiseuille flow, as shown in Fig. 6.

Let us consider the static case with no gas flowing in which the direction of the temperature gradient along the channel is opposite to that of the pressure gradient and dynamical equilibrium is established, that is, the case where the flow rate induced by the pressure gradient is equal to the opposite flow by the thermal stress. In this case the temperature gradient is surely considered to be supported by an applied pressure gradient. The ratio of the temperature gradient to the pressure gradient from the present analysis is shown in Fig. 7. The ratio approaches 0.5 as $\delta \rightarrow 0$. This was already pointed out by Knudsen, and is called thermal transpiration. The ratio takes a relatively large value even in the transition region. It becomes smaller as the inverse Knudsen increases, and approaches zero as $\delta$ $\rightarrow \infty$.

The dashed line in Fig. 7 demonstrates the curve obtained from the experiment by Edmonds and Hobson $^{5)}$. The present result is qualitatively related to the experimental curve, but shows slightly higher value.

As described above, the Poiseuille flow and the thermal creep have different parabolic velocity profiles

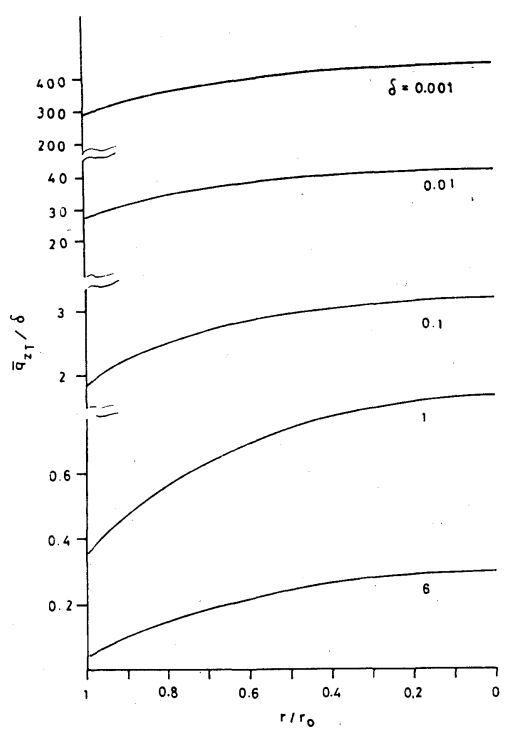

Fig. 6 Gas velocity profile of the thermal creep

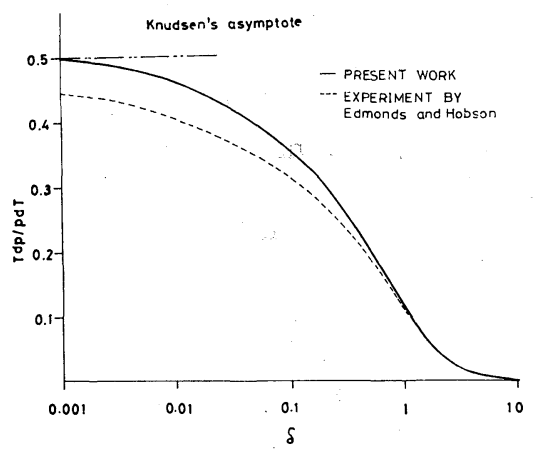

Fig. 7 Relationship of the thermal transpiration with the inverse Knudsen number

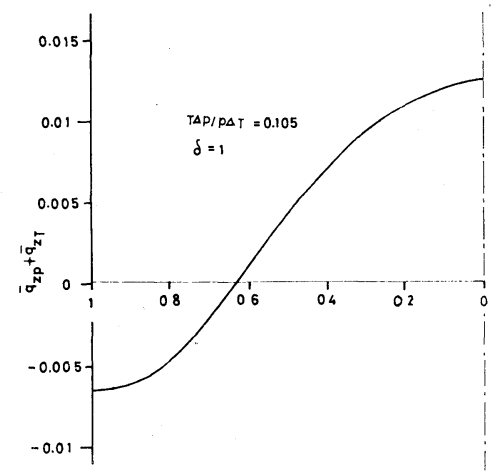

Fig. 8 Gas velocity profile under the condition of no gross flow

from each other, but not even ones. Accordingly, even if no gross flow rate occurs, the local gas velocity at arbitrary $r$-ordinate normal to the flow direction is not necessarily equal to zero. The gas velocity profile for the case where the equilibrium is established at $\delta=1$ is shown in Fig. 8. The figure demonstrates that when the state of dynanfical equilibrium is established, the gas is creeping steadily along the channel wall and is at the same time flowing back through the center of the channel.

As a practical example of thermal transpiration, the problem of measurement of the pressure of cooled gas with a McLeod gauge will be given here. We consider 
the case where a vessel in which air is held is kept at $-194^{\circ} \mathrm{C}$ by liquid air. The cold trap lying between the gas vessel and McLeod gauge is kept at the same temperature, and the McLeod gauge is settled at room temperature of $20^{\circ} \mathrm{C}$. If then the gauge is connected directly with the cold trap by a circular tube having $10 \mathrm{~mm}$ inner diameter, it indicates a pressure of $0.001 \mathrm{mmHg}$. In this case, $T=\left(T_{1}+T_{2}\right) / 2=186^{\circ} \mathrm{K}$ $\Delta T=214^{\circ} \mathrm{K}$, and if we choose for the mean pressure $p=0.0008$, we have $\delta=0.0928$ and the ratio $T \Delta p \mid p \Delta T=$ 0.35 (from Fig. 7). Then, the pressure difference due to creep is $0.000403 \mathrm{mmHg}$, and the true pressure of the gas is considered to be $0.000597 \mathrm{mmHg}$. And the mean pressure $p=\left(p_{1}+p_{2}\right) / 2=0.000799 \mathrm{mmHg}$, which is nearly equal to the first-tried pressure $p=0.0008$. (If the error is great, we have to repeat the calculation above.)

\section{Conclusion}

In the analysis of the Boltzmann-Krook equation for the rarefied gas flow problem, the iteration approach which uses the continuum solution as the first guess, as offered in this paper, directly leads us to the solution. Convergence is relatively fast in speed in the continuum-slip, in the transition, and even in the free molecular region. This approach is notably easy to handle in calculation, compared with the Chapman-Enskog method, Gross and others' BGK half-range moment method, and so on.

The present solution for the gas flow induced by pressure gradient gives appropriate results which are in fair agreement with the experimental data. The simplicity of the solution may provide convenience for practical purposes.

The present analysis also appropriately predicts the relationship of the flow rate of thermal creep with the inverse Knudsen number, and makes it clear that thermal creep has a profound effect on rarefied-gas channel flow in the slip, in the transition, and especially in the free molecular flow region. In the treatment of rarefied gas flow at a nonuniform temperature, therefore, the effect of thermal creep has to be taken into account.

A detailed discussion of thermal creep has not been given here for want of experimental study. It now remains an open question to clarify the behavior of thermal creep from the experimental point of view.

\section{Appendix}

\section{Free molecular solution for the Poiseuille flow}

It is considered that the gas velocity has an even profile and that it is independent of the inverse Knudsen number. Therefore, we can write

$$
\underset{\substack{\operatorname{q}_{z P}=0 \\ \lim \dot{\delta} \rightarrow 0}}{ }(=\text { constant })
$$

for the free molecular limit. Substituting Eq. (A-1) into Eq. (15) of the text as the first guess, we have

$$
\bar{q}_{z P}=\frac{1}{\pi^{3 / 2}} \int_{\Sigma} \int_{0}^{\infty} \int_{-\infty}^{\infty}\left[2 \delta \bar{\xi}_{z}^{2} c_{1}-\bar{\xi}_{z}^{2}\right]
$$

$$
\frac{\exp \left\{-\left[\frac{\delta}{\xi_{p}}\left(\left|\overline{\boldsymbol{r}}-\overline{\boldsymbol{r}}^{\prime}\right|\right)+\bar{\xi}_{p}^{2}+\bar{\xi}_{z}^{2}\right]\right\}}{\left|\overline{\boldsymbol{r}}-\overline{\boldsymbol{r}}^{\prime}\right|} d \bar{\xi}_{z} d \bar{\xi}_{p} d \Sigma
$$

After integration of Eq.(A-2) with respect to $\bar{\xi}_{p}, \bar{\xi}_{z}$, and $\sum$, the gas velocity can be written as

$$
\bar{q}_{z P}(\bar{r})=\frac{1}{2 \pi} \int_{0}^{2 \pi}\left(2 c_{1}-1 / \delta\right)\left[1 / 2-T_{1}(\chi)\right] d \psi
$$

The transcendental function $T_{1}(\chi)$ for small $\chi$ takes the form:

$$
\underset{\delta \rightarrow 0}{T_{1}}(\chi)=\frac{1}{2}-\frac{\pi^{1 / 2}}{2} \chi-\chi^{2} \ln \chi / 2
$$

Using Eq.(A-4), and integrating Eq.(A-3) with respect to $\psi$, the gas velocity becomes

$$
\bar{q}_{z P}(\bar{r})=\frac{1}{\pi^{1 / 2}} E(\bar{r}, \pi / 2)+\frac{\delta \ln \delta}{2}
$$

where $E(r, \theta)$ is the ellipsoidal function of first order defined by

$$
E(\bar{r}, \theta)=\int_{0}^{\theta}\left(1-\bar{r}^{2} \sin ^{2} t\right)^{1 / 2} d t
$$

The volume flow rate is then

$$
\bar{Q}_{P}=\int_{0}^{1} 2 \pi \bar{r} \bar{q}_{z P}(\bar{r}) d \bar{r}=\frac{4 \pi^{1 / 2}}{3}+\frac{\pi}{2} \delta \ln \delta
$$

\section{Free molecular solution for the thermal creep}

According to Knudsen's free molecular solution, the gas velocity for the free molecular limit can be expressed as

$$
\bar{q}_{z T}=c_{2}
$$

Substituting Eq.(A-7) into Eq.(16) of the text as the first guess, we have

$$
\begin{aligned}
\bar{q}_{z T}(\bar{r})= & \frac{3}{\pi^{3 / 2}} \int_{\Sigma} \int_{0}^{\infty} \int_{-\infty}^{\infty}\left[2 \delta \bar{\xi}_{z}^{2} c_{2}-\bar{\xi}_{z}^{2}\left(\bar{\xi}_{z}^{2}+\bar{\xi}_{p}^{2}-5 / 2\right)\right] \\
& \cdot \frac{\exp \left\{-\left[\delta\left(\left|\overline{\boldsymbol{r}}-\overline{\boldsymbol{r}}^{\prime}\right|\right) / \xi_{p}+\bar{\xi}_{p}^{2}+\bar{\xi}_{z}^{2}\right]\right\}}{\left|\overline{\boldsymbol{r}}-\overline{\boldsymbol{r}}^{\prime}\right|} d \bar{\xi}_{z} d \bar{\xi}_{p} d \Sigma
\end{aligned}
$$

After carrying out the integration, the result is

$$
\bar{q}_{z T}(\bar{r})=\frac{1}{2 \pi \delta} \int_{0}^{2 \pi}\left[-T_{3}(\chi)+T_{1}(0)+T_{3}(\chi)-T_{1}(\chi)\right] d \psi
$$

The function $T_{1}(\chi)$ and $T_{3}(\chi)$ for small $\delta$ can be written as

$$
\begin{aligned}
& T_{1}(\chi)=\frac{1}{2}-\frac{\pi^{1 / 2}}{2} \chi-\frac{1}{2} \chi^{2} \ln \chi \\
& T_{3}(\chi)=\frac{1}{2}-\frac{\pi^{1 / 2}}{4} \chi+\frac{1}{4} \chi^{2}+\frac{\pi^{1 / 2}}{12} \chi^{3}-\frac{\chi^{4}}{24} \ln \chi
\end{aligned}
$$

Using the property of the functions, the gas velccity becomes

$$
\bar{q}_{z T}(\bar{r})=\frac{1}{2 \pi^{1 / 2}} E(r, \pi / 2)+\frac{1}{2} \delta \ln \delta
$$

The volume flow rate is

$$
Q_{T}=\frac{2 \pi^{1 / 2}}{3}+\frac{\pi}{2} \delta \ln \delta
$$

\section{Acknowledgment}

We wish to thank Professor Y. Matsumura for his kind assistance.

\section{Nomenclature}

$$
\begin{aligned}
& c_{1}, c_{2}=\text { constant } \quad[-] \\
& f \quad=\text { velocity distribution function of the gas molecules } \\
& \begin{array}{lll}
f_{0} & =\text { Maxwellian distribution function } & {\left[\mathrm{sec}^{3} / \mathrm{cm}^{3}\right]} \\
\left.\mathrm{sec}^{3} / \mathrm{cm}^{3}\right]
\end{array} \\
& q_{z} \quad=\text { gas velocity in the } z \text {-direction }\left(q_{z p}\right. \text { for Poiseuille } \\
& \text { flow, } q_{z T} \text { for thermal creep) } \\
& \bar{q}_{z P} \quad=\text { nondimensional gas velocity defined by } \\
& \bar{q}_{z P}=q_{z P} / \kappa_{P}(2 k T / m)^{1 / 2} \\
& \bar{q}_{z T} \quad=\text { nondimensional gas velocity defined by } \\
& \bar{q}_{z T}=q_{z T} / \kappa_{T}(2 k T / m)^{1 / 2} \\
& Q_{P} \quad=\text { volume flow rate of the flow induced by the } \\
& \text { pressure gradient } \\
& Q_{T} \quad=\text { volume flow rate of the thermal creep } \quad\left[\mathrm{cm}^{3} / \mathrm{sec}\right] \\
& =\text { nondimensional flow rate defined by } \\
& \bar{Q}_{P}=Q_{P} / r_{o}^{3}(2 k T / m)^{1 / 2} d p / p d z \\
& \bar{Q}_{T} \quad=\text { nondimensional flow rate defined by } \\
& \bar{Q}_{T}=Q_{T} / r_{o}^{3}(2 k T / m)^{1 / 2} d T / T d z \\
& =\text { radial ordinate }
\end{aligned}
$$




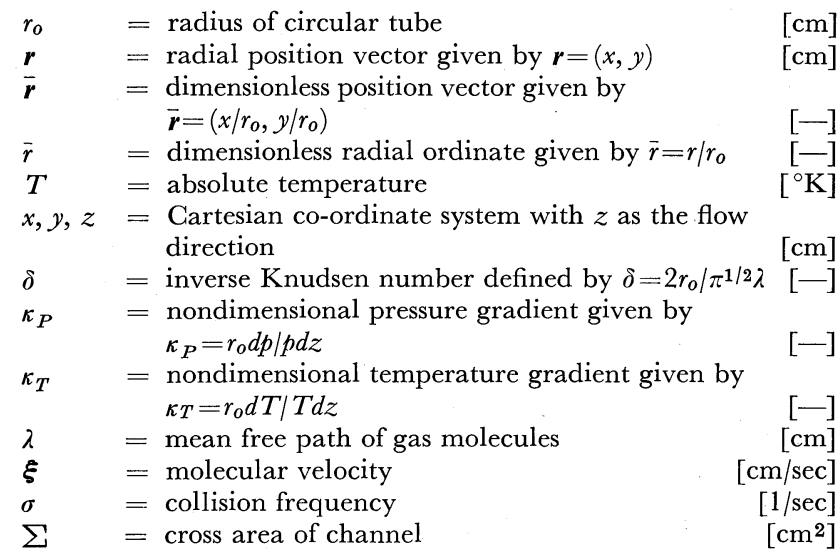

\section{Literature Cited}

1) Adzumi, H.: Bull. Chem. Soc. Japan, 12, 285 (1937)
2) Bosanquet, C. H.: British TA Report, BR-507, 9, 127 (1944)

3) Cercignani, C. and F. Sernagiotto: Phys. Fluids, 9, 40 (1966)

4) Dong, W.: University of California Radiation Laboratory Report UCRL-3353 (1956)

5) Edmonds, T. and J. F. Hobson: J. Vac. Sci. Technol., 2, 182 (1965)

6) Gross, E. P., E. A. Jackson and S. Ziering: Ann. Phys., 1, 141 (1957)

7) Iuchi, S. and T. Kanki: Kagaku Kōgaku, 34, 607 (1970)

8) Kanki, T. and S. Iuchi: Phys. Fluids, 16, 695 (1973)

9) Kanki, T. and S. Iuchi: Kagaku Kögaku, 36, 442 (1972)

10) Kennard, E. H.: "Kinetic Theory of Gases", p. 331, McGraw Hill Book Company, Inc., New York (1938)

11) Knudsen, M.: Ann. Phys., 28, 75 (1909)

12) Liu, C. Y.: Phys. Fluids, 11, 481 (1968)

13) Nanba, S., H. Shinagawa and S. Kawamura: Kagaku Kōgaku, 35, 592 (1971)

14) Sone, Y. and K. Yamamoto: Phys. Fluids, 11, 1672 (1968)

15) Scott, D. S. and F. A. L. Dullien, : AIChE J., 3, 293 (1962)

16) Takao, K.: Trans. Japan Soc. Aerospace Sci., 4, 82 (1961)

17) Willis, D. R.: Phys. Fluids, 5, 127 (1962)

\title{
PULSATING TURBULENT FLOW IN A TUBE*
}

\author{
TOKuro MIZUSHINA, Toshiro MARUYAMA \\ AND YASumi SHIOZAKI** \\ Department of Chemical Engineering, Kyoto University, \\ Kyoto, Japan
}

\begin{abstract}
Pulsating turbulent flow in a tube was studied experimentally. Measured instantaneous profiles of the velocity and the intensity of turbulence can be clearly classified into two groups by means of a certain critical period of pulsation. At longer periods of pulsation, the shapes of the velocity profiles are similar to those for steady flow, while the intensity of turbulent fluctuation does not pulsate; whereas at shorter periods, the velocity profiles are not similar to those for steady flow, while the intensity of turbulent fluctuation pulsates oppositely to the velocity.

From measuring the time intervals between bursts in both steady and pulsating turbulent flows, it was clarified that the range of burst periods occurring in pulsating flow was the same as the preferred range of burst periods in steady flow. If the pulsation period is longer than the upper limit of this range, the mean burst period is equal to that of steady flow, but if the pulsation period is included in the range of burst periods for steady flow, the bursting of the same period as the pulsation period dominates.
\end{abstract}

\section{Introduction}

Pulsating tube flow has been used in several practical situations, for example, when fluid is pumped through a channel by a reciprocating device. We will consider a general case of a periodically pulsating flow with a mean value which is different from zero.

Pulsating laminar flow was studied analytically by Sexl and by Szymanski. For the corresponding turbulent flow, however, there have been only a few experi-

* Received on May 30, 1973

Presented in part at the 6th Autumn Meeting of The Soc. of Chem. Engrs., Japan, Dec. 29, 1972

** Sumitomo Chemical Co., Ltd.

于606 京都市左京区吉田本町

京都大学工学部化学工学教室 丸山敏朗 mental studies, because it is very difficult to study a turbulent structure that varies during the cycle of pulsation. Qualitatively, some features have been shown. Schultz-Grunow? indicated that velocity profiles for pulsating turbulent flow are similar to the profiles for steady flow through a gradually convergent or divergent pipe. Recently, Gerrard ${ }^{2)}$ suggested that the turbulence intensity diminishes during acceleration, and that in decelerating flow the turbulence intensity increases.

The objective of this study is to clarify the essential mechanism for a systematic treatment of pulsating turbulent flow. Whereas the periodicity of bursting is essential in steady turbulent flow, both periodicity of bursting and that of pulsation must be taken into account with regard to each other in pulsating turbulent flow; it is believed that an investigation of the 